\title{
NOTES
}

\section{Regulation of Pore-size Distribution of Poly( $\gamma$-methyl L-glutamate) Spheres as a Gel Permeation Chromatography Packings ${ }^{\dagger}$}

\author{
Chuichi Hirayama, * Hirotaka Ihara, Shoji Nagaoka \\ Hideki Furusawa, and Shinji Tsuruta \\ Department of Applied Chemistry, Faculty of Engineering, \\ Kumamoto University, Kumamoto 860, Japan
}

(Received January 4, 1990)

\begin{abstract}
KEY WORDS Polypeptide / Poly( $\gamma$-methyl L-glutamate) / Porous Beads / Gel Permeation Chromatọgraphy / Liquid Chromatography /
\end{abstract}

We have reported that porous spheres prepared from poly( $\gamma$-methyl L-glutamate) (PMLG) alone without any other support showed a high flow-rate resistance and amphiphilicity as aqueous gel permeation chromatography (GPC) packings. ${ }^{2-4}$ These favorable properties are related to the formation of secondary structures of peptide chains due to inter- and intramolecular hydrogen bondings. The sphering and macroreticulation of PMLG spheres were achieved using the "suspension and evaporation" technique ${ }^{4}$ previously reported and the pore-size was controlled by adjusting a kind or amount of diluent added in the sphering process. ${ }^{4,5}$ However, we have often observed that the spherical shape could not be maintained, thus reducing the number of theoretical plates, when diluent as a macroreticulating agent was used in large amounts to prepare macroporous packings. It is closely related that effective diluents are poor solvents for PMLG.

In this study, we succeeded in macroreticulating the PMLG beads with a large exclusion molecular weight $\left(10^{5}-10^{7}\right)$, narrow pore-size distribution, and surprisingly high flow-rate resistance. These beads are quite useful for

† Polypeptide Spheres VI. See ref 1.

* To whom all correspondence should be addressed. some affinity chromatographies. ${ }^{5,6}$

\section{EXPERIMENTAL}

\section{Sphering Procedure}

$75 \mathrm{ml}$ of $9.5 \mathrm{wt} \%$ PMLG (degree of polymerization, 350) solution, prepared by polymerization of $N$-carboxyanhydride of $\gamma$-methyl L-glutamate in 1,2-dichloroethane (DCE), was diluted with $325 \mathrm{ml}$ of a DCE solution containing $19.2 \mathrm{ml}$ of di(2-ethylhexyl)phthalate (DEHP). The solution was put in a $500 \mathrm{ml}$ autoclave, and heated at $100^{\circ} \mathrm{C}$ for $1-48 \mathrm{~h}$. After cooling to room temperature, the solution was mixed with $400-4000 \mathrm{ml}$ of a $0.2-3.5 \mathrm{wt} \%$ solution of aqueous poly(vinyl alcohol) (PVA, Gohsenol GH-20) and suspended by stirring at $45^{\circ} \mathrm{C}$ for $24-48 \mathrm{~h}$. During this time, the DCE was gradually evaporated from the suspension particles and PMLG spheres obtained were washed successively with water, hot water and methanol to form macroporous PMLG spheres. Figure 1 shows typical optical and electron micrographs of PMLG spheres obtained. 

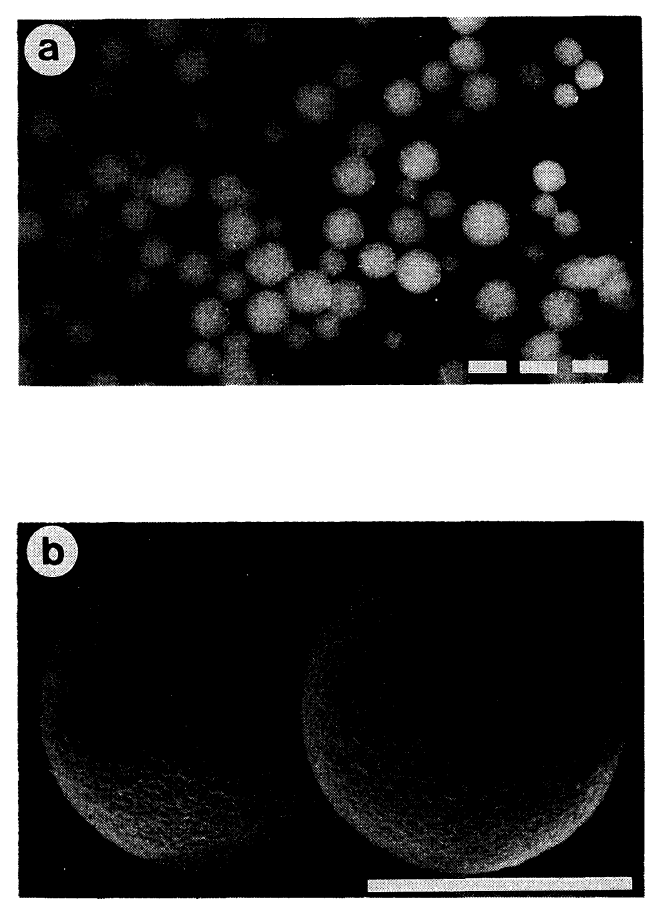

Figure 1. Optical (a) and electron (b) micrographs of typical porous PMLG spheres (p-9), scale bars present $50 \mu \mathrm{m}$.

\section{Measurement}

The distribution of pore-size of PMLG spheres was estimated by the exclusion molecular weight $\left(M_{\text {lim }}\right)$ and the porosity, which were determined from the calibration curves of an aqueous GPC.

The degased aqueous slurry of packings which diameters of $44-105 \mu \mathrm{m}$ was packed into a glass column $(30 \times 0.5 \mathrm{~cm}$ i.d. $)$ at a pressure of $2.0 \mathrm{~kg} \mathrm{~cm}^{-2}$. The pumps used were Waters Model 510 and JASCO 880-PU, and the detector used was the Shodex refracto monitor SE-51. A $20 \mu$ portion of the sample prepared in an aqueous solution $\left(5.0 \mathrm{mg} \mathrm{ml}^{-1}\right)$ was injected through a Rheodyne Model 7125 injector. The calibration curves were prepared with use of blue dextran $\left(M_{n} 2.00 \times 10^{6}\right)$, a homologous pullulan $\left(M_{n} 8.53 \times 10^{5}, 3.80 \times\right.$ $10^{5}, 1.86 \times 10^{5}, 1.00 \times 10^{5}, 4.80 \times 10^{4}, 2.37 \times$ $10^{4}$, and $5.80 \times 10^{3}$ ) maltoheptaose, maltohexaose, maltotriose, maltose, and $\mathrm{D}_{2} \mathrm{O}$. The
$M_{\text {lim }}$ value was obtained by extrapolating the linear part of the curve. ${ }^{4}$ The porosity is given by eq. $1 .^{4}$ This value corresponds to the elution volume of $\mathrm{D}_{2} \mathrm{O}$ which is the smallest molecule permeable within the reticulated matrix of PMLG spheres.

$$
\text { Porosity }(\%)=\frac{V_{\mathrm{D}_{2} \mathrm{O}}-V_{0}}{V_{\mathrm{t}}-V_{0}} \times 100
$$

where $V_{\mathrm{t}}, V_{0}$, and $V_{\mathrm{D}_{2} \mathrm{O}}$ are the total volume of the column, the interstitial volume of the gel bed, and the elution volume of $\mathrm{D}_{2} \mathrm{O}$, respectively. Equation 1 is transformed into eq. 2 , because the elution volume of permeable substances with molecular weights from $M_{i}$ to $M_{i+1}$ is corresponds to $V_{i+1}-V_{i}$.

$$
\text { Porosity }(\%)=\frac{\sum_{i=1}^{n-1}\left(V_{i+1}-V_{i}\right)}{V_{\mathrm{t}}-V_{0}} \times 100
$$

where $V_{1}$ and $V_{n}$ correspond to $V_{0}$ anf $V_{\mathrm{D}_{2} \mathrm{O}}$ in eq 1 , respectively. The pore-size distribution is estimated by plotting $100 \times\left(V_{i+1}-V_{i}\right) /\left(V_{\mathrm{t}}-\right.$ $V_{0}$ ) against the molecular weight of permeable substances $\left(M_{i}\right.$ to $\left.M_{i+1}\right)$.

Secondary structures of PMLG were estimated by FT-IR spectroscopy (Perkin-Elmer 1640) and polarimeter (Horiba Sepa-200).

\section{RESULTS AND DISCUSSION}

In general, the pore-size of macroporous resins has been estimated by mercury penetration, and this technique is useful for dry resins. However, the pore-size in dry resins does not always correspond to that of wet resins, especially in a hydrophilic gel. Therefore we applied a gel permeation chromatography (GPC) technique to estimate the pore-size distribution of PMLG spheres.

It was found that the pore-size distribution was notably affected by incubation time of PMLG solution, concentration of PVA in a suspension medium, and volume ratio of suspension medium to PMLG solution in the 
Table I. Values of $M_{\text {lim }}$ and porosity of PMLG spheres prepared with the "suspension and evaporation" technique

\begin{tabular}{|c|c|c|c|c|c|}
\hline \multirow{2}{*}{$\begin{array}{c}\text { Sphere } \\
\text { No. }\end{array}$} & \multirow{2}{*}{$\begin{array}{l}\text { Incubation } \\
\text { time } / \mathrm{h}^{\mathrm{a}}\end{array}$} & \multicolumn{2}{|c|}{ Suspension medium } & \multirow{2}{*}{$\begin{array}{l}M_{1 \mathrm{im}}^{\mathrm{e}} \\
\times 10^{-4}\end{array}$} & \multirow{2}{*}{$\frac{\text { Porosity }}{\%}$} \\
\hline & & $w t \%^{b}$ & Ratio $^{c}$ & & \\
\hline p-1 & 0 & 2.5 & 3 & $3 \sim 6$ & $73 \sim 74$ \\
\hline $\mathrm{p}-2$ & 1 & ", & $"$ & $20 \sim 40$ & $82 \sim 83$ \\
\hline $\mathrm{p}-3$ & 6 & , & , & $25 \sim 45$ & $81 \sim 83$ \\
\hline $\mathrm{p}-4$ & 12 & , & $"$ & $20 \sim 30$ & $82 \sim 84$ \\
\hline p-5 & 24 & , & , & $35 \sim 50$ & $81 \sim 83$ \\
\hline p-6 & 48 & $"$ & ", & $40 \sim 50$ & $83 \sim 85$ \\
\hline p-7 & 6 &, & 1 & $15 \sim 30$ & $76 \sim 77$ \\
\hline $\mathrm{p}-8$ & , & $"$ & 2 & $10 \sim 25$ & $75 \sim 79$ \\
\hline p-9 & , & $"$ & 6 & $150 \sim 230$ & $>81$ \\
\hline $\mathrm{p}-10$ & $"$ & $"$ & 10 & $150 \sim 230$ & $>81$ \\
\hline $\mathrm{p}-11$ & , & 0.2 & 6 & $15 \sim 25$ & $79 \sim 81$ \\
\hline p-12 & , & 0.5 & $"$ & $18 \sim 22$ & $77 \sim 80$ \\
\hline $\mathrm{p}-13$ & , & 1.5 &, & $200 \sim 250$ & $>82$ \\
\hline p-14 & $"$ & 3.5 & ", & $150 \sim 230$ & $>86$ \\
\hline
\end{tabular}

a Incubated at $100^{\circ} \mathrm{C}$.

b Concentration of PVA.

c The volume ratio of suspension medium to PMLG.

d Exclusion molecular weight calibrated using polysaccharides.

e Estimated by eq 1 .

sphering process. Table I summarizes the values of $M_{\text {lim }}$ and porosity determined by the calibration curve of an aqueous GPC.

Figure 2 shows typical calibration curves of aqueous GPC for the PMLG spheres prepared with incubation at $100^{\circ} \mathrm{C}$ and without incubation. The $M_{\text {lim }}$ and porosity increased from 40000 to 300000 and from 73 to $82 \%$, respectively by incubation of the PMLG solution at $100^{\circ} \mathrm{C}$. In addition, the incubation narrowed the pore-size distribution, as shown in Figure $2 b$. The addition of di(2-ethylhexyl)phthalate (DEHP) to PMLG solution induces partial inhomogenization, causing the spheres to become misshapen and lowering chromatographic resolution in the GPC system, because the diluent is a poor solvent for PMLG. Therefore, we assumed that the incubation at $100^{\circ} \mathrm{C}$ promoted a homogenization of diluent domains to produce macroporous PMLG spheres with a narrow pore-size distribution. This assumption is supported by examining $[\eta]$ value of material PMLG solution. It was confirmed that the value of $[\eta]$ decreased from 2.45 to 1.95 with decrease of specific rotation $[\alpha]$ by incubating for more than $12 \mathrm{~h}$, as shown in Figure 3. Since the values of $[\eta]$ and $[\alpha]$ no longer lowered by further incubation, the decrease of these values is not due to decomposition of peptide chains. Presumably, the incubation promotes the disassociation of PMLG in the material solution. In general, such association of PMLG is produced during polymerization of $N$-carboxyanhydride or induced by addition of a poor solvent such as DEHP, and is related to the formation of $\beta$-structure. In the case of the sphering of PMLG without incubation, the PMLG spheres contained partially $\beta$-structural chains (amide $\mathrm{V}, 700 \mathrm{~cm}^{-1}$ ). On the other hand, $\beta$-structure was not detected for the PMLG spheres prepared with incubation. According to FT-IR spectra, the PMLG spheres prepared with incubation was almost constructed by $\alpha$-helical peptide chains (amide $\mathrm{V}, 620 \mathrm{~cm}^{-1}$ ). Therefore, it is considered that the decrease 

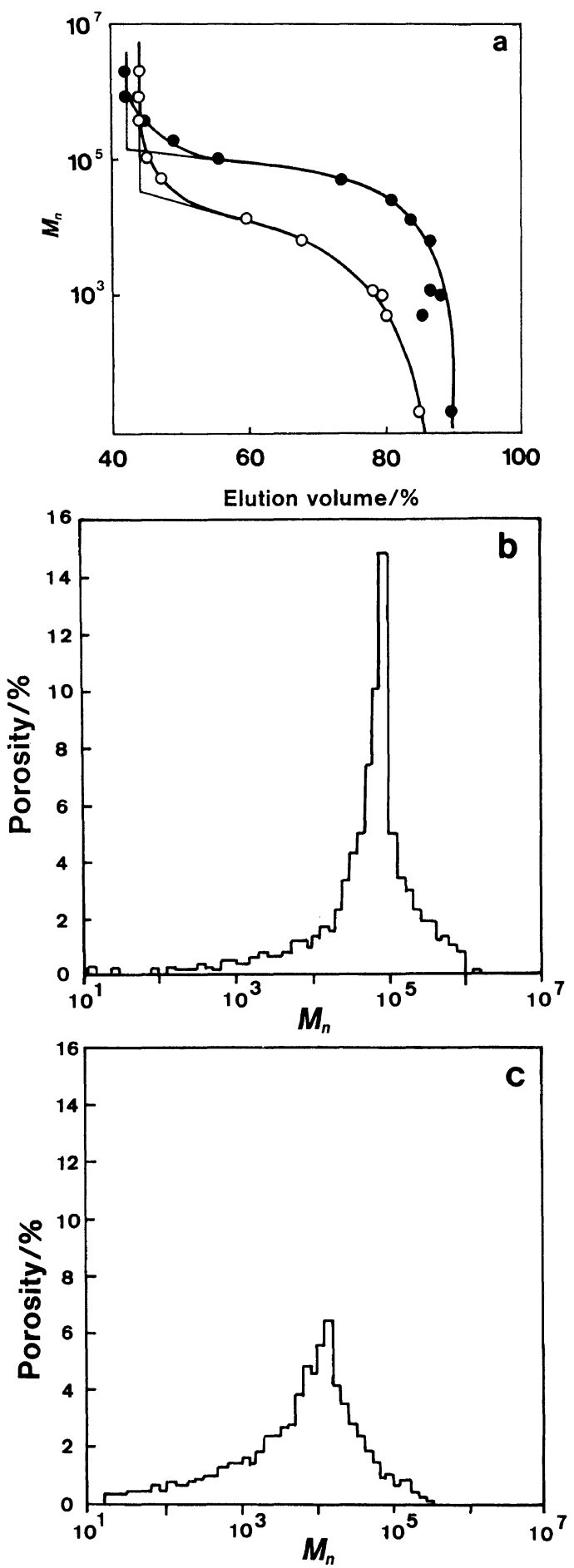

Figure 2. Calibration curves of GPC (a) and pore-size distribution ( $b$ and $c$ ) for porous PMLG spheres. a) - p-3; - $\bigcirc-, \mathrm{p}-1$; b) p-3; c) p-1.

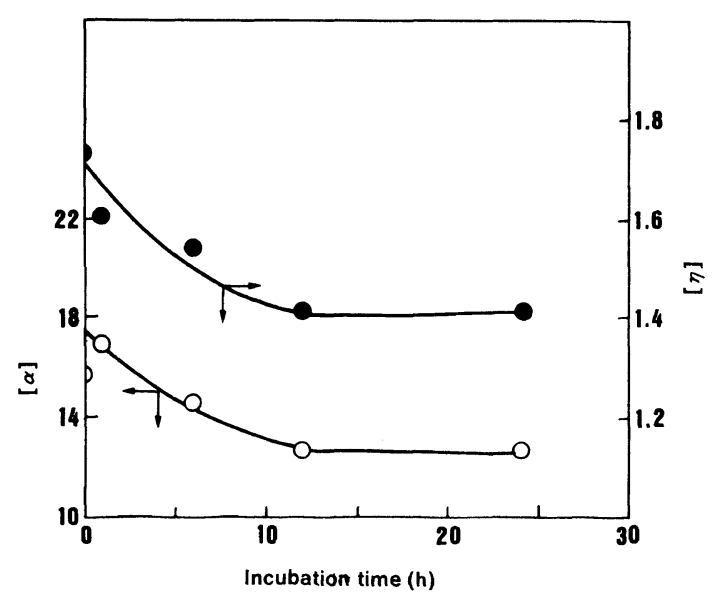

Figure 3. Relationships between incubation time $\mathrm{e}^{\mathrm{a}}$ and values of $[\eta]$ and $[\alpha]$ in the PMLG solution. ${ }^{\mathrm{b} a}$ At $100^{\circ} \mathrm{C}$. $2 \mathrm{ml} \mathrm{g}^{-1}$ of di(2-ethylhexyl)phthalate is contained in $2 \mathrm{wt} \%$ dichloroethane solution of PMLG.

of $[\alpha]$ value with incubation indicates the conformational change from $\beta$-structure to $\alpha$-helix (or random coil).

High resolution GPC must be realized by spherical packings with narrow pore-size distribution. The resolution in the GPC mode was examined. As shown in Figure 4, the column packed with the $\mathrm{p}-3$ spheres gave a good resolution compared with that of the p-1 spheres. There is no significant difference in the theoretical plate number, and the molecular size of permeable substances chosen are within those of the effective fractionation for each columns. Therefore, the difference of resolution in Figure 4 is attributable to that of pore-size distribution.

In addition, it was found that the $M_{\text {lim }}$ was remarkably depending upon the volume ratio of the suspension medium to the PMLG solution (suspension ratio) and the PVA concentration of the suspension medium. For example, it increased from $1.6 \times 10^{5}$ to above $2 \times 10^{6}$ or $2.2 \times 10^{5}$ to $2.0 \times 10^{6}$ with the increase of the suspension ratio from 2 to 6 or the concentration of PVA from 0.2 to $1.5 \mathrm{wt} \%$, respectively as shown in Table I. It is considered that the suspension ratio and concentration of 


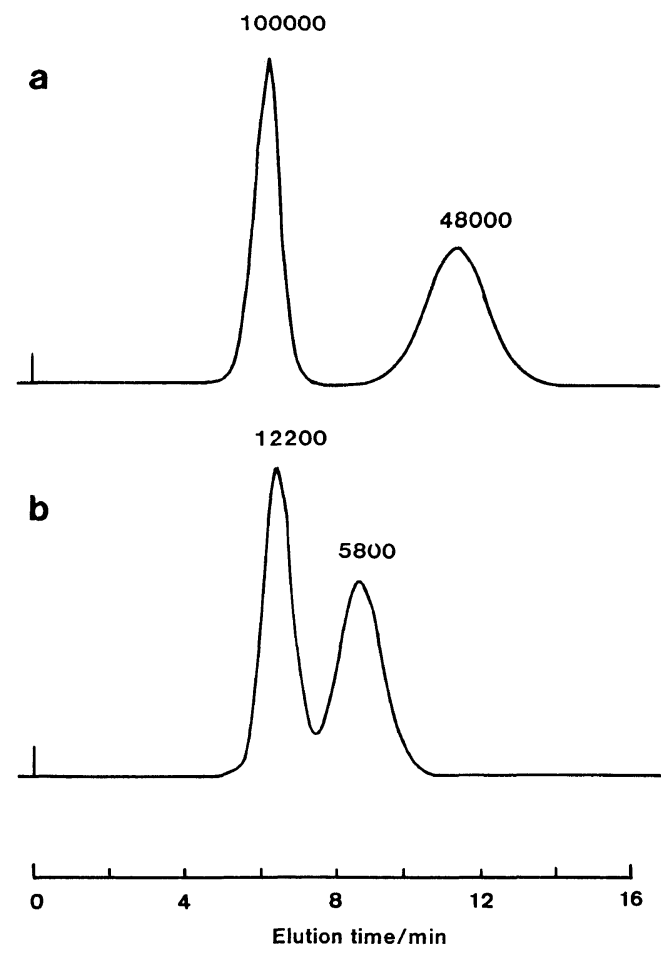

Figure 4. Typical gel permeation chromatograms in the p-3 (a) and the p-1 (b) columns. Flow-rate, $0.5 \mathrm{ml} \mathrm{min}^{-1}$; detection, refractive index; column, $300 \times 5 \mathrm{~mm}$ i.d.; eluent, distilled water. The number in the figure shows the molecular weight of standard pullulans.

PVA are concerned in the stabilization of suspension particles.

In conclusion, we succeeded in preparing macroporous PMLG beads with a large $M_{\text {lim }}$ and narrow pore-size distribution by modifying the "suspension and evaporation" technique. Since the technique makes effective use of a small amount of diluent as a macroreticulating agent, the PMLG particles obtained maintain good spherical shape regardless of the $M_{1 \mathrm{im}}$ value. In addition, the PMLG spheres tolerate high flow-rates in aqueous GPC because of rigidity due to hydrogen bondings peculiar to polypeptides as shown in Figure 5. Although we have previously reported that the macroporous glucomannan spheres showed higher flow-rate resistance than any other aqueous packing from polysaccharides, ${ }^{8-13}$ the PMLG

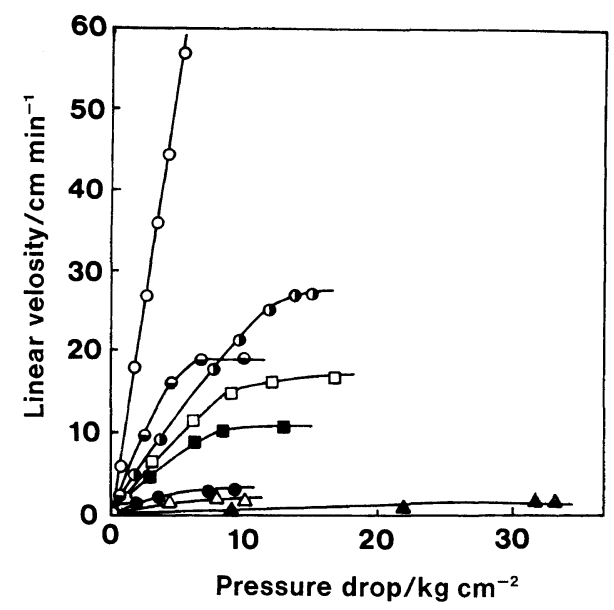

Figure 5. Relationships between flow-rate and pressure drop in aqueous GPC. All measurements were carried out using columns packed at the same conditions. - $\mathrm{O}-$ p-9, PMLG ( $\left.M_{\text {lim }} 1500000\right) ;-\bigcirc-$ glucomannan $^{8,9}$ (2000000); - - - cellulose $^{10}\left(M_{\mathrm{lim}} 800000\right)$; - - pullulan $^{11,12}\left(M_{\text {lim }} 1000000\right)$; - $\square-$ dextran $^{13}$ (commercially available Sephacryl S-400, $\left.M_{\lim } 500000\right)$; - $\square$ dextran ${ }^{13}$ (commercially available Sephacryl S-300, $M_{\text {lim }}$ 200000); $-\triangle$ 一, dextran ${ }^{13}$ (commercially available

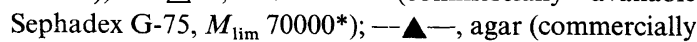
available Sepharose 4B, $M_{\text {lim }} 3000000^{*}$ ).

* Value indicated by manufactured literature.

spheres showed even further resistance.

These favorable properties of PMLG packings will enable application to some affinity chromatographies. For example, we confirmed that functional groups could be introduced with ease by using reactive residual carbomethoxy groups of PMLG. ${ }^{7}$ The PMLG spheres and their derivatives have unique affinities for proteins ${ }^{5}$ and endotoxins. ${ }^{6}$ And their resolution must be directly related to the pore-size distribution. These applications are under investigating.

Acknowledgments. We thank Mr. T. Shono for his capable assistance. This work was partially supported by a Grant-in-Aid for Scientific Research from the Ministry of Education, Science, and Culture of Japan. 


\section{Polypeptide Spheres}

\section{REFERENCES}

1. Polypeptide Spheres VI: C. Hirayama, H. Ihara, and K. Tanaka, J. Chromatogr., 450, 271 (1988).

2. H. Ihara, T. Yoshinaga, Y. Motozato, and C. Hirayama, Polym. J., 17, 1301 (1985).

3. C. Hirayama and H. Ihara, J. Chromatogr., 347, 357 (1985).

4. H. Ihara, T. Yoshinaga, and C. Hirayama, J. Chromatogr., 362, 197 (1986).

5. H. Ihara, H. Furusawa, X. Li, and C. Hirayama, $J$. Appl. Polym. Sci., in prints.

6. C. Hirayama, H. Ihara, X. Li, K. Aihara, and T. Tsunoda, Polym. Prepr., Jpn., 38, 2220 (1989); C. Hirayama, H. Ihara, and X. Li, J. Chromatogr. Biomed. Appl., to be submitted.
7. C. Hirayama and H. Ihara, Polym. Appl., 37, 16 (1988).

8. C. Hirayama, H. Ihara, M. Nakamura, Y. Motozato, and T. Kunitake, J. Chromatogr., 409, 175 (1987).

9. Y. Motozato, H. Ihara, M. Nakamura, M. Shiba, and C. Hirayama, Nippon Kagaku Kaishi, 61 (1986).

10. H. Furusawa, unpublished data in this laboratory.

11. Y. Motozato, H. Ihara, T. Tomoda, and C. Hirayama, J. Chromatogr., 355, 434 (1986).

12. Y. Motozato, H. Ihara, T. Tomoda, and C. Hirayama, Nippon Kagaku Kaishi, 1187 (1986).

13. The $M_{\text {lim }}$ values were determined under the same packing and elution procedures as those of the PMLG beads. The values determined were smaller than the $2 \times 10^{6}$ and $7.5 \times 10^{5}$ showed in the manufactured literature. 